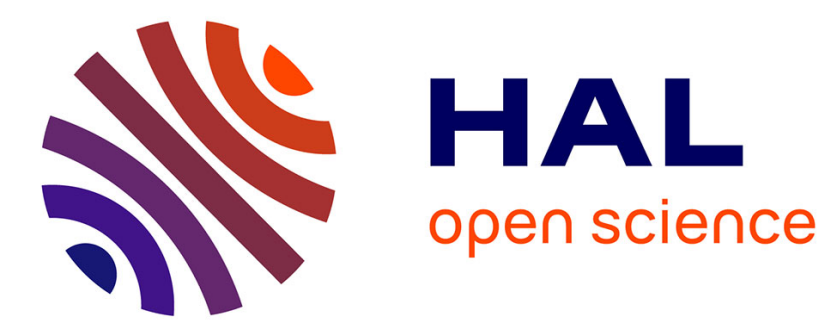

\title{
Etude de films magnétiques de l'alliage Fe60A140
}

\author{
A. Fnidiki, J. Eymery
}

\section{To cite this version:}

A. Fnidiki, J. Eymery. Etude de films magnétiques de l'alliage Fe60Al40. Journal de Physique I, 1991, 1 (6), pp.953-968. 10.1051/jp1:1991179 . jpa-00246379

\section{HAL Id: jpa-00246379 https://hal.science/jpa-00246379}

Submitted on 1 Jan 1991

HAL is a multi-disciplinary open access archive for the deposit and dissemination of scientific research documents, whether they are published or not. The documents may come from teaching and research institutions in France or abroad, or from public or private research centers.
L'archive ouverte pluridisciplinaire HAL, est destinée au dépôt et à la diffusion de documents scientifiques de niveau recherche, publiés ou non, émanant des établissements d'enseignement et de recherche français ou étrangers, des laboratoires publics ou privés. 
Classification

Physics Abstracts

$75.50 \mathrm{~B}-76.80-81.40 \mathrm{R}$

\title{
Etude de films magnétiques de l'alliage $\mathrm{Fe}_{60} \mathrm{Al}_{40}$
}

\author{
A. Fnidiki (*) et J. P. Eymery \\ Laboratoire de Métallurgie Physique (**), 40, avenue du Recteur Pineau, F-86022 Poitiers \\ Cedex, France
}

(Reçu le 10 décembre 1990, accepté sous forme définitive le 11 février 1991)

\begin{abstract}
Résumé. - On montre que la technique de coévaporation permet, comme l'implantation ionique, d'obtenir $\mathrm{Fe}_{60} \mathrm{Al}_{40}$ désordonné au point de vue atomique et ferromagnétique à $293 \mathrm{~K}$. Il s'avère cependant qu'il y a des différences entre l'alliage massif implanté et les films minces de même composition en ce qui concerne l'évolution en température des propriétés magnétiques. Dans le premier cas, il y a disparition des effets magnétiques dès $400 \mathrm{~K}$; dans le second, on observe encore une fraction ferromagnétique importante après chauffage et retour à l'ambiante. De plus, le champ hyperfin au noyau du fer 57 croît, ce qui conduit à l'hypothèse de zones enrichies en fer et, par suite, indique que l'alliage ne se réordonne pas.
\end{abstract}

\begin{abstract}
We show that the coevaporation technique like ion implantation, allows us to obtain $\mathrm{Fe}_{60} \mathrm{Al}_{40}$ which is both chemically disordered and ferromagnetic at $293 \mathrm{~K}$. However, differences between implanted bulk alloy and thin films of the same composition have been observed concerning the temperature evolution of magnetic properties. In the first case, magnetic effects disappear as soon as $400 \mathrm{~K}$; in the second case, one still observes a significant ferromagnetic fraction at room temperature after annealing at $700 \mathrm{~K}$ and cooling down. Moreover, the hyperfine field at the 57 iron nucleus increases. This leads to the hypothesis of iron rich phases and hence indicates that the alloy does not reorder.
\end{abstract}

\section{Introduction.}

Dans le domaine de concentration $20 \leqslant x \leqslant 50$, les alliages $\mathrm{Fe}_{100}{ }_{x} \mathrm{Al}_{x}$ cristallisés peuvent présenter la surstructure $\mathrm{DO}_{3}$ pour $x<32$ et la surstructure $\mathrm{B} 2$ pour $x>32$. En outre, les propriétés magnétiques de ces alliages dépendent fortement de la présence ou de l'absence d'ordre atomique. Le diagramme de phases magnétiques des alliages ordonnés est reproduit sur la figure 1 ; on retiendra surtout, pour la zone B2, la présence d'une phase paramagnétique à l'ambiante et celle d'une phase mictomagnétique à basse température [1]. Le comportement magnétique des mêmes alliages à l'état désordonné s'avère au contraire radicalement différent puisqu'ils sont de type ferromagnétique sur tout le domaine $20 \leqslant x \leqslant 50[2,3]$. Dans le cas du type B2 qui nous intéresse ici, le désordre atomique ne peut

(*) Present address : Département de Physique. Faculté des Sciences, F-76130 Mont Saint-Aignan, France.

(**) U.R.A. 131 du C.N.R.S. 
être obtenu que par des traitements mécaniques extrêmes (broyage, limage) qui réduisent le matériau en poudre.

Les résultats de la littérature, considérés dans leur ensemble, indiquent que les propriétés magnétiques d'un atome de fer, dans le système Fe-Al, sont déterminées en premier lieu par la nature de ses premiers voisins. D'après la référence [4], le moment magnétique d'un atome de fer varie, en fonction du nombre de premiers voisins $\mathrm{Fe}$ et $\mathrm{Al}$, comme l'indique la figure 2 ; il s'avère que le moment reste constant jusqu'à deux atomes d'Al, puis décroît d'environ $4 \%$ pour $3 \mathrm{Al}$ et enfin tend rapidement vers 0 entre 3 et $5 \mathrm{Al}$. On conçoit alors aisément que les alliages de la zone $\mathrm{B} 2$ deviennent ferromagnétiques à l'ambiante lorsqu'on les désordonne puisqu'on accroît alors la probabilité de liaisons Fe-Fe. Des études effectuées par effet Mössbauer en transmission ont effectivement montré l'existence d'interactions hyperfines magnétiques [4, 5]. Plus récemment, Shiga et Nakamura [6] ont apporté quelques précisions supplémentaires grâce à un ajustement des spectres Mössbauer par une distribution de champs hyperfins. La distribution montre alors deux pics distincts dans la gamme 0-300 kOe et les auteurs concluent à l'existence d'un nombre critique $n_{\mathrm{c}}$ de premiers voisins Fe pour donner lieu à des interactions magnétiques; ils proposent: $n_{\mathrm{c}} \sim 4$. De même, une étude effectuée sur l'alliage $\mathrm{Fe}_{65} \mathrm{Al}_{35}$ limé a conduit à des résultats analogues [7].

Au cours d'études publiées entre 1983 et 1987 , nous avons montré que le désordre atomique pouvait aussi être induit dans l'alliage $\mathrm{Fe}_{60} \mathrm{Al}_{40}$ par implantation ionique [8-12]. En particulier, l'implantation d'ions $\mathrm{Al}^{2+}$ à $293 \mathrm{~K}$ avec une énergie de $260 \mathrm{keV}$ s'est avérée produire une couche ferromagnétique de $220 \mathrm{~nm}$ d'épaisseur à la surface du matériau massif qui ne subissait ainsi aucune destruction. Rappelons brièvement qu'en utilisant la technique de l'effet Mössbauer en réflexion qui consiste à capter les électrons de conversion et Auger (CEMS), les résultats suivants ont pu être obtenus pour la dose de $3 \times 10^{14}$ ions $/ \mathrm{cm}^{2}$ : champ

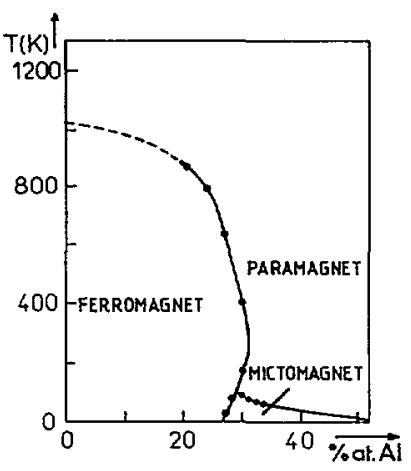

Fig. 1.

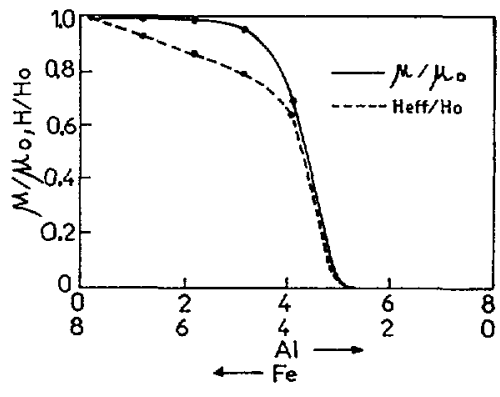

Fig. 2.

Fig. 1. - Diagramme de phases magnétiques des alliages $\mathrm{Fe}-\mathrm{Al}$ en fonction de la concentration d'aluminium. D'après la référence [1].

[Magnetic phase diagram of $\mathrm{Fe}-\mathrm{Al}$ alloys as a function of aluminium concentration. From reference [1].]

Fig. 2. - Variations du moment magnétique et du champ effectif en fonction du nombre de premiers voisins $\mathrm{Fe}$ et $\mathrm{Al}$ d'un atome de fer dans les alliages Fe-Al $\left(\mu_{0}=2,2 \mu_{\mathrm{B}}\right.$ et $\left.H_{0}=330 \mathrm{kOe}\right)$. D'après la référence [4].

[Variations in magnetic moment and effective field as a function of the number of $\mathrm{Fe}$ and $\mathrm{Al}$ nearest neighbours of an iron atom in Fe-Al alloys $\left(\mu_{0}=2.2 \mu_{\mathrm{B}}\right.$ and $\left.H_{0}=330 \mathrm{kOe}\right)$. From reference [4].] 
hyperfin moyen $\bar{H}=211 \mathrm{kOe}$, fraction paramagnétique d'atomes de fer $f_{\mathrm{p}}=33 \%$, température critique de disparition de l'éclatement magnétique hyperfin $\sim 400 \mathrm{~K}$. Dans le but de préciser les propriétés magnétiques de la couche implantée, une étude de résonance ferromagnétique a également été effectuée; elle a permis de déterminer, à $293 \mathrm{~K}$, l'aimantation spécifique $\sigma=86 \mathrm{uem} / \mathrm{g}$ et le facteur de Landé $g=2,06$.

Outre l'écrouissage et l'implantation ionique, une troisième technique vient de se révéler capable de produire $\mathrm{Fe}_{60} \mathrm{Al}_{40}$ à la fois désordonné au point de vue atomique et ferromagnétique à $293 \mathrm{~K}$; il s'agit de la coévaporation. Dans une récente note, nous avons montré que les films minces ainsi déposés sur un support de kapton présentaient cette propriété [13]. Le but du présent article est de faire la synthèse des résultats obtenus ultérieurement tant sur l'état structural que sur les propriétés magnétiques de films $\mathrm{Fe}_{60} \mathrm{Al}_{40}$ préparés par coévaporation et d'effectuer des comparaisons avec l'alliage massif de même composition implanté à l'ambiante. L'état structural des films sera étudié par microscopie électronique à transmission M.E.T., microanalyse X et SIMS (Secondary Ion Mass Spectroscopy). Les propriétés magnétiques seront déterminées en premier lieu par effet Mössbauer en transmission et en réflexion (CEMS) et en second lieu par des mesures d'aimantation réalisées sur un magnétomètre à échantillon vibrant et un dispositif d'effet Kerr.

\section{Techniques expérimentales.}

Les films ont été préparés par coévaporation dans un évaporateur Riber qui est essentiellement constitué des éléments suivants :

- un système de vide à pompe ionique et sublimateur de titane permettant d'obtenir dans les meilleures conditions un vide de $10^{-10}$ Torr ;

- un système d'évaporation à chauffage par bombardement électronique constitué de deux canons Airco-Temescal d'une puissance de $8 \mathrm{~kW}$ pilotés par une seule alimentation; film.

- deux quartz permettant de contrôler la vitesse de dépôt ainsi que l'épaisseur totale du

La fabrication par coévaporation des échantillons destinés à la présente étude a été réalisée dans un vide de $10^{-8}$ Torr et avec une vitesse d'évaporation de chaque constituant de l'ordre de 0,2 à $0,3 \mathrm{~nm} / \mathrm{s}$. Les techniques d'investigation ont évidemment conditionné le choix du support et de l'épaisseur des films. C'est ainsi que des échantillons de $100 \mathrm{~nm}$ déposés sur $\mathrm{NaCl}$ ont permis de réaliser les études en microscopie électronique et microanalyse $\mathrm{X}$; de même des films de 100, 200 et $250 \mathrm{~nm}$ d'épaisseur déposés soit sur kapton, soit sur silicium monocristallin $\{111\}$, ont été utilisés pour l'effet Mössbauer en réflexion et l'effet Kerr. De tels films seront qualifiés par la suite de «films minces ». Au contraire, les films qualifiés "d'épais» auront une épaisseur d'un micron; de tels films ont été utilisés pour l'effet Mössbauer en transmission et les mesures au magnétomètre et ont toujours été déposés sur un support de kapton. Enfin, les expériences de SIMS ont été réalisées aussi bien sur des films minces que sur des films épais.

Les observations en microscopie électronique ont été faites sur un microscope à transmission classique (Philips EM300) fonctionnant sous une tension de $100 \mathrm{kV}$. La détermination de la composition des films a été effectuée par microanalyse $\mathrm{X}$ principalement avec un détecteur à diode $\mathrm{Si}-\mathrm{Li}$ associé à un microscope électronique JEOL $200 \mathrm{CX}$ fonctionnant sous une tension de $200 \mathrm{kV}$. Pour les films déposés sur kapton, la composition a été également mesurée par microanalyse $\mathrm{X}$ en utilisant cette fois une microsonde de Castaing de marque Cameca.

L'analyse SIMS a été réalisée avec un dispositif expérimental ISA-RIBER LAS 3000 ; elle a utilisé $\mathrm{Xe}^{+}$comme ion primaire avec une incidence de $45^{\circ}$, une énergie de $5 \mathrm{keV}$ et une 
intensité de courant de $20 \mathrm{nA}$. Les profils de plusieurs ions ont été obtenus en fonction de l'épaisseur pulvérisée: ${ }^{56} \mathrm{Fe}^{+},{ }^{57} \mathrm{Fe}^{+},{ }^{27} \mathrm{Al}^{++}$et aussi ${ }^{12} \mathrm{C}^{+}$et ${ }^{16} \mathrm{O}^{+}$pour estimer la quantité de contamination en carbone et en oxygène au cours de l'évaporation.

Concernant la technique CEMS, les spectres d'électrons ont été obtenus avec une source de cobalt 57 dispersée dans une matrice de rhodium $(50 \mathrm{mCi})$, un compteur proportionnel à flux gazeux $\mathrm{He}+5 \% \mathrm{CH}_{4}$ et un spectromètre Mössbauer conventionnel; le compteur a été récemment décrit en détail dans la référence [14]. Rappelons que la technique CEMS permet d'analyser la surface des matériaux et les films minces; son efficacité est maximale sur la première centaine de nanomètres et moindre sur la deuxième. En géométrie de transmission, nous avons également fait usage d'un cryostat MERIC pour les études à basse température. Pour l'ajustement des spectres, nous avons utilisé un programme de calcul permettant une analyse des spectres Mössbauer en distribution de champs hyperfins basée sur la méthode de Window [15].

Les mesures d'aimantation ont été réalisées sur un magnétomètre à échantillon vibrant et un dispositif d'effet Kerr; la longueur d'onde du laser utilisé dans celui-ci était de 632,8 nm. La première technique a été appliquée aux films épais et la seconde aux films minces; dans ce dernier cas, seulement quelques dizaines de nanomètres sont analysés. Les mesures magnétiques ont été effectuées en appliquant un champ extérieur, soit parallèlement, soit perpendiculairement à la surface des échantillons étudiés. Ces dernières ont été réalisées au Laboratoire de Magnétisme de Bellevue.

\section{Etat structural des films $\mathrm{Fe}_{60} \mathrm{Al}_{40}$ après coévaporation.}

L'état structural a été étudié par microscopie électronique à transmission, microanalyse $\mathrm{X}$ et SIMS. La microanalyse $X$ a permis de montrer que la composition exacte des films correspondait à $\mathrm{Fe}_{59,4} \mathrm{Al}_{40,6}$ et que les résultats étaient reproductibles. La microscopie électronique révèle des anneaux de diffraction (voir Fig. 3a) caractéristiques d'un alliage cristallin, cubique centré et désordonné chimiquement car aucun anneau de surstructure n'est visible. La figure $3 b$ est une image en champ sombre réalisée avec la réflexion (110). Celle-ci montre que la couche observée est constituée de petits grains. La taille des plus petits est de l'ordre de $5 \mathrm{~nm}$ tandis que celle des plus gros avoisine $50 \mathrm{~nm}$; la moyenne de la distribution se situe autour de $15-20 \mathrm{~nm}$. L'étude en SIMS a été effectuée sur un film d'épaisseur $1 \mu \mathrm{m}$
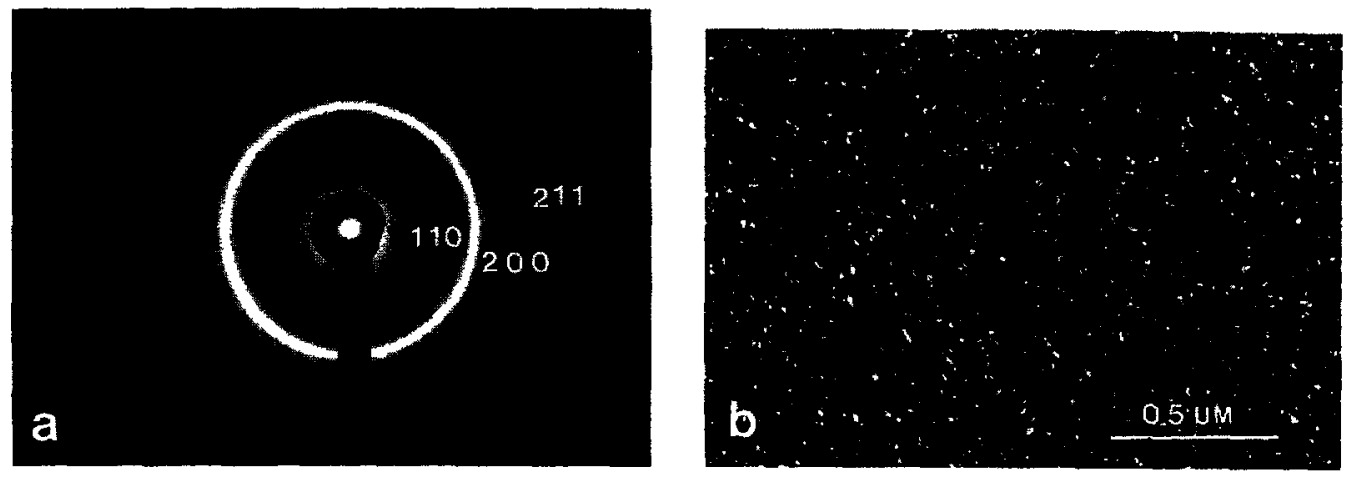

Fig. 3. - Film de $100 \mathrm{~nm}$ coévaporé : a) cliché de diffraction et b) image en champ sombre réalisée avec la réflexion (110).

[Coevaporated $100 \mathrm{~nm}$ film : a) electron diffraction pattern and b) dark field image taken with the (110) reflection.] 
déposé sur kapton. Elle a permis de détecter des oscillations de concentration de longue période perpendiculaires au plan du film. Les profils des ions $\mathrm{Fe}^{+}$et $\mathrm{Al}^{3+}$ sont représentés sur la figure 4 ; on peut constater que les maxima de $\mathrm{Fe}$ correspondent aux minima d'Al et vice-versa. De telles fluctuations de concentration ont une amplitude inférieure ou égale à $6 \%$; ce résultat sera intéressant pour discuter à la fois les grandeurs et les variations de certaines propriétés magnétiques. De plus, il est connu qu'il peut exister en outre des fluctuations locales de composition liées à la technique de coévaporation car la vitesse d'évaporation de chaque source n'est pas rigoureusement constante; toutefois, le SIMS ne peut mettre en évidence ces dernières. Enfin, on constate la présence de contaminations en carbone et en oxygène, mais leur importance n'apparaît pas critique.

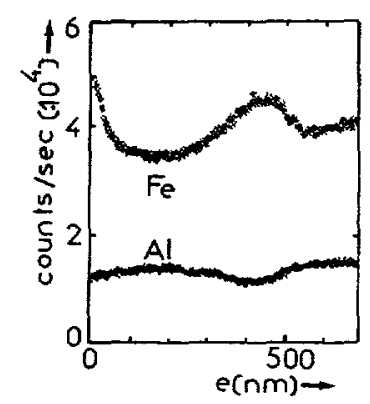

Fig. 4. - Profils SIMS de Fe et Al dans le cas d'un film d'épaisseur $1 \mu \mathrm{m}$.

[SIMS depth profiling of $\mathrm{Fe}$ and $\mathrm{Al}$ in the case of a $1 \mu \mathrm{m}$ thick film.]

\section{Propriétés magnétiques des films minces $\mathrm{Fe}_{60} \mathrm{Al}_{40}$.}

\section{ETUDE PAR LA TECHNIQUE CEMS.}

Echantillons déposés sur kapton. - Le spectre d'électrons obtenu à $293 \mathrm{~K}$ sur un film de $250 \mathrm{~nm}$ déposé sur kapton, est représenté sur la figure $5 \mathrm{a}$. Il se compose d'une raie centrale paramagnétique et d'un sextuplet élargi. En ce qui concerne ce dernier, seules les raies 1 et 6 sont clairement visibles; les raies 2 et 5 apparaissent faibles, ce qui indique que la polarisation des spins est plus proche de la normale que du plan du film. Le spectre a été dépouillé par un programme de calcul qui permet d'analyser le sextuplet en distribution de champs hyperfins et d'ajuster la raie centrale à une raie lorentzienne. Pour la raie paramagnétique, les paramètres suivants ont été déterminés : déplacement isomérique $I S=+0,10 \mathrm{~mm} / \mathrm{s} \mathrm{par}$ rapport au fer $\alpha$, effet quadripolaire $Q S=0 \mathrm{~mm} / \mathrm{s}$ et largeur à mi-hauteur $\Gamma=0,37 \mathrm{~mm} / \mathrm{s}$. La composante magnétique a été dépouillée en utilisant 50 sextuplets élémentaires de largeur égale à la largeur naturelle et des valeurs de champs comprises entre 200 et $300 \mathrm{kOe}$; les paramètres suivants ont été déterminés : $I S=+0,05 \mathrm{~mm} / \mathrm{s}, Q S=0 \mathrm{~mm} / \mathrm{s}$, intensité relative des raies 2 et 5 des sextuplets élémentaires $b=0,8$ (ce qui implique que l'orientation des spins est de $35^{\circ}$ par rapport à la normale au film). La distribution du champ hyperfin $P(H)$ est montrée sur la figure 6. L'allure de $P(H)$ est pratiquement symétrique par rapport à la valeur moyenne $\vec{H}=250 \mathrm{kOe}$; la déviation standard $\sigma_{H}$ est de $18 \mathrm{kOe}$. Enfin, le pourcentage de fer ferromagnétique est de $30 \%$.

Il apparaît ainsi une première différence entre les propriétés des films minces et celles des échantillons massifs $\mathrm{Fe}_{60} \mathrm{Al}_{40}$ implantés. Elle concerne la valeur du champ hyperfin moyen à 


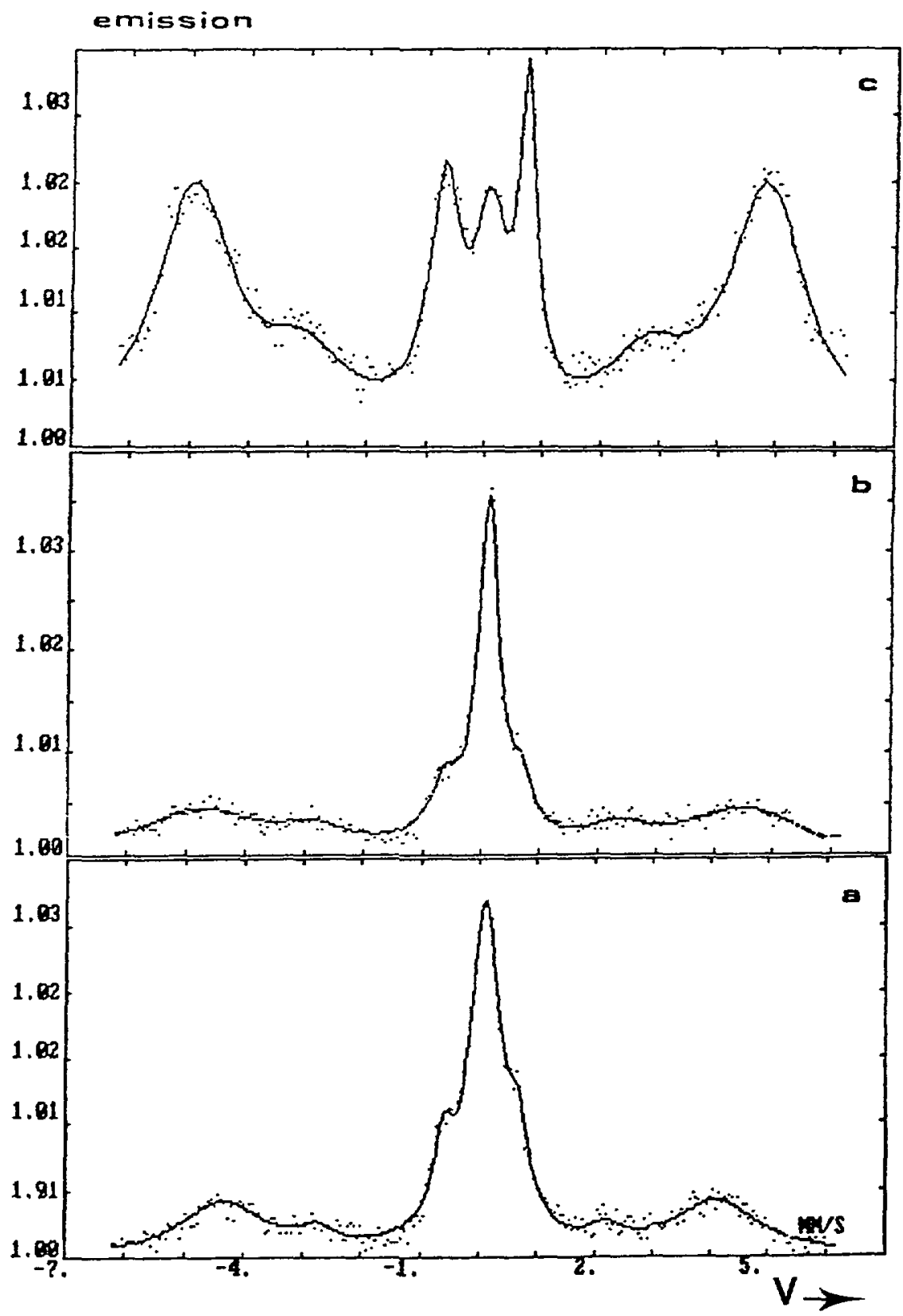

Fig. 5. - Spectres CEMS obtenus sur un film de $250 \mathrm{~nm}$ déposé sur kapton : a) état coévaporé ; b) recuit à la température de $493 \mathrm{~K}$; c) $693 \mathrm{~K}$.

[CEM spectra taken from a $250 \mathrm{~nm}$ film deposited onto a kapton substrate : a) as-coevaporated state ; b) annealed at $493 \mathrm{~K}$; c) $693 \mathrm{~K}$.]

la température ambiante qui est de $250 \mathrm{kOe}$ dans le premier cas et de $211 \mathrm{kOe}$ dans le second cas. Cette différence peut être partiellement interprétée par les fluctuations de composition révélées par l'étude SIMS ; elle sera discutée ultérieurement au paragraphe 6. 


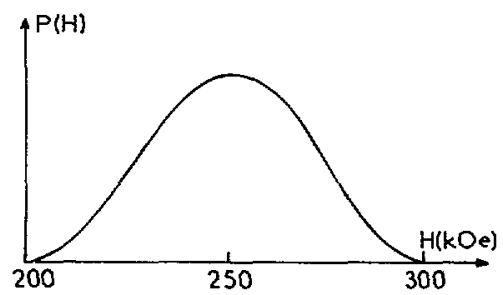

Fig. 6. - Distribution du champ hyperfin de la fraction de fer ferromagnétique obtenue à partir de la figure 5 a.

[Hyperfine field distribution of the iron ferromagnetic fraction for spectrum (a) in figure 5.]

On va maintenant exposer les résultats concernant l'évolution de l'alliage en fonction de la température. L'échantillon étudié par CEMS a été recuit 1 heure sous vide de $10^{-6}$ Torr à la température désirée puis les spectres d'électrons réalisés à $293 \mathrm{~K}$. Les figures $5 \mathrm{~b}$ et c représentent les spectres obtenus avec un échantillon recuit à 493 puis $693 \mathrm{~K}$. Les paramètres Mössbauer obtenus par un dépouillement analogue au précédent sont récapitulés dans le tableau I dans lequel figurent également ceux de l'échantillon brut de coévaporation. On constate que le champ hyperfin moyen $\bar{H}$ et la fraction de fer ferromagnétique croissent quand la température croît. En particulier, pour la température de $693 \mathrm{~K}, f_{\mathrm{f}}$ et $\bar{H}$ s'accroissent respectivement jusqu'à $80 \%$ et $300 \mathrm{kOe}$. Ce résultat, qui indique vraisemblablement la croissance de zones enrichies en fer, sera également discuté dans le paragraphe 6 . Il apparaît donc que l'alliage $\mathrm{Fe}_{60} \mathrm{Al}_{40}$ préparé par coévaporation ne se réordonne pas du point de vue chimique, ce qui constitue encore une différence entre les échantillons coévaporés d'une part et massifs implantés d'autre part.

Tableau I. - Paramètres Mössbauer des spectres CEMS obtenus à $293 \mathrm{~K}$ avec un film coévaporé sur kapton. La partie gauche correspond à la fraction de fer ferromagnétique et celle de droite à la fraction paramagnétique $\left(\bar{H}=\right.$ champ hyperfin moyen, $\sigma_{H}=$ déviation standard, $b=$ intensité relative des raies 2 et $5, f_{\mathrm{f}}=$ fraction ferromagnétique, $I S=$ déplacement isomérique par rapport au fer $\alpha$ et $\Gamma=$ largeur à mi-hauteur).

[Mössbauer parameters of CEM spectra taken at $293 \mathrm{~K}$ from a film deposited onto a kapton substrate. Left and right sides correspond to ferromagnetic and paramagnetic iron fractions respectively $\left(\bar{H}\right.$ is the average hyperfine field, $\sigma_{H}$, the standard deviation, $b$, the relative intensity of lines 2 and $5, f_{\mathrm{f}}$, the ferromagnetic fraction, $I S$, the isomer shift with respect to $\alpha$ iron, and $\Gamma$, the half linewidth).]

\begin{tabular}{|l|cccc|cc|}
\hline \multicolumn{1}{|c|}{$T(\mathrm{~K})$} & $\bar{H}(\mathrm{kOe})$ & $\sigma_{H}(\mathrm{kOe})$ & $b$ & $f_{\mathrm{f}}(\%)$ & $I S(\mathrm{~mm} / \mathrm{s})$ & $\Gamma(\mathrm{mm} / \mathrm{s})$ \\
\hline 293 & 250 & 18 & 0,8 & 28 & 0,10 & 0,37 \\
après 1 h à 493 & 265 & 23 & 0,7 & 30 & 0,10 & 0,23 \\
après 1 h à 693 & 300 & 36 & 0,4 & 80 & 0,10 & 0,4 \\
\hline
\end{tabular}

Echantillons déposés sur silicium. - En ce qui concerne le comportement thermique des films minces, les investigations précédentes ont été limitées par le support de kapton; c'est 


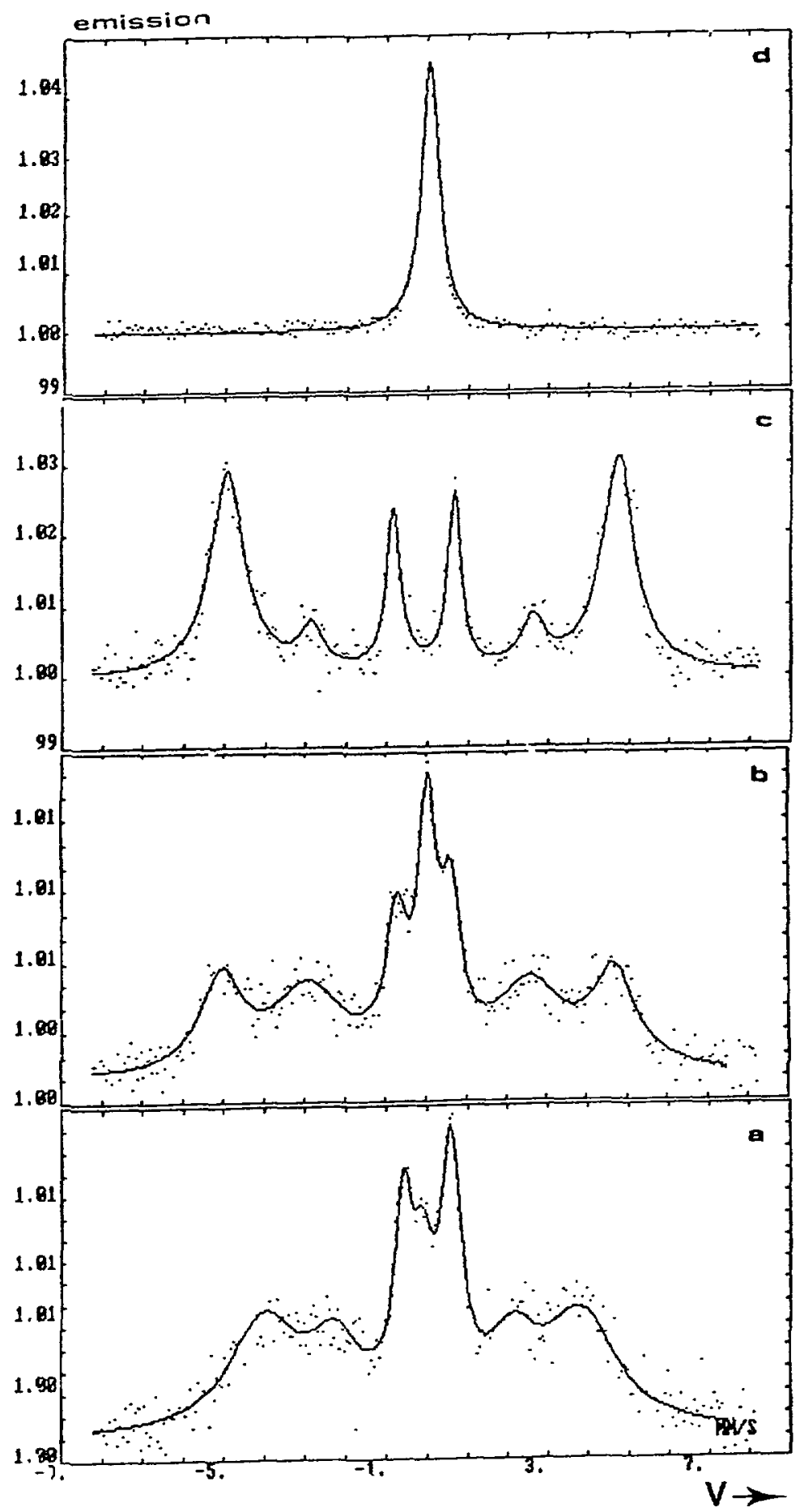

Fig. 7. - Spectres CEMS obtenus sur un film de $200 \mathrm{~nm}$ déposé sur $\mathrm{Si}:$ a) après déposition ; b) après recuit $1 \mathrm{~h}$ à $573 \mathrm{~K}$; c) $773 \mathrm{~K}$; d) $873 \mathrm{~K}$.

[CEM spectra taken from a $200 \mathrm{~nm}$ film deposited onto a Si substrate : a) as-deposited state; b) after one hour annealing at $573 \mathrm{~K}$; c) $773 \mathrm{~K}$; d) $873 \mathrm{~K}$. 
pourquoi, dans un second̀ temps, on a changé de support et utilisé un substrat de silicium. Les propriétés magnétiques à $293 \mathrm{~K}$ ont été caractérisées par la technique CEMS et par l'effet Kerr sur un film d'épaisseur $200 \mathrm{~nm}$ déposé sur silicium et ensuite l'étude du comportement thermique a été poursuivie par CEMS. Enfin, concernant l'état structural, une étude complémentaire par SIMS a été effectuée à $293 \mathrm{~K}$ sur le même échantillon recuit à $1073 \mathrm{~K}$.

Le spectre d'électrons à $293 \mathrm{~K}$ de l'échantillon coévaporé est représenté sur la figure 7 . Le dépouillement de ce spectre a conduit à un champ hyperfin moyen $\bar{H}$ égal à $218 \mathrm{kOe}$ et à une fraction ferromagnétique égale à $74 \%$ (voir Tab. II). Ces résultats sont cette fois en bon accord avec ceux obtenus sur les échantillons massifs implantés [9] ou broyés [4].

Tableau II. - Paramètres Mössbauer des spectres CEMS obtenus à $293 \mathrm{~K}$ avec un film coévaporé sur silicium. Les notations employées sont les mêmes que celles de la légende du tableau 1.

[Mössbauer parameters of CEM spectra taken at $293 \mathrm{~K}$ from a film deposited onto a Si substrate. Symbols are the same as those of the caption of table I.]

\begin{tabular}{|l|cccc|cc|}
\hline \multicolumn{1}{|c|}{$T(\mathrm{~K})$} & $\bar{H}(\mathrm{kOe})$ & $\sigma_{H}(\mathrm{kOe})$ & $b$ & $f_{\mathrm{f}}(\%)$ & $I S(\mathrm{~mm} / \mathrm{s})$ & $\Gamma(\mathrm{mm} / \mathrm{s})$ \\
\hline 293 & 218 & 51 & 1,1 & 74 & 0,15 & 0,5 \\
après 1 h h̀̀ 573 & 290 & 18 & 2 & 46 & 0,10 & 0,5 \\
après 1 h à̀ 773 & 3,1 & 15 & 0,45 & 100 & - & - \\
après 1 h hà 873 & - & - & - & - & 0,15 & 0,26 \\
\hline
\end{tabular}

On a également complété la connaissance des propriétés magnétiques en mesurant la rotation Kerr polaire $\theta_{\mathrm{K}}$. Rappelons que l'effet Kerr ne permet d'atteindre que la surface des films analysés. La figure 8 montre la variation de $\theta_{\mathrm{K}}$ en fonction du champ magnétique appliqué perpendiculairement à la surface de l'échantillon. On constate que la saturation a lieu pour $\theta_{\mathrm{K}}=13^{\prime}$ et $4 \pi M=6,4 \mathrm{kOe}$. En outre, en attribuant à l'alliage une densité de 6,02 on trouve que l'aimantation spécifique $\sigma$ est de $84,5 \mathrm{uem} / \mathrm{g}$. Ce résultat est également en bon accord avec la valeur obtenue par résonance ferromagnétique sur les échantillons massifs implantés [12].

A partir des données ci-dessus, il est tout à fait satisfaisant de noter que les trois modes de préparation de l'alliage $\mathrm{Fe}_{60} \mathrm{Al}_{40}$ désordonné (broyage, implantation et coévaporation)

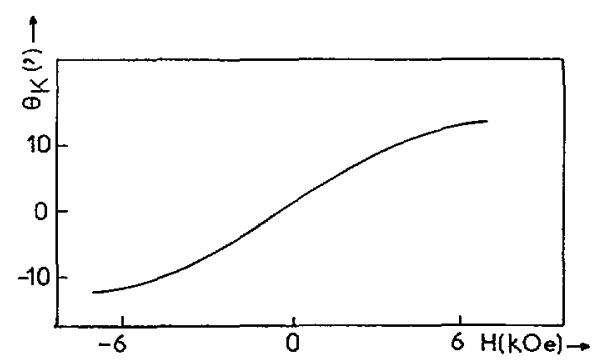

Fig. 8. - Rotation Kerr polaire $\theta_{K}$ en fonction du champ magnétique appliqué à $293 \mathrm{~K}$.

[Kerr rotation angle $\theta_{K}$ versus applied magnetic field at $293 \mathrm{~K}$.] 
conduisent presque aux mêmes propriétés magnétiques à l'ambiante sauf dans le cas du support kapton. La technique de coévaporation apparaît donc être une nouvelle méthode pour préparer l'alliage $\mathrm{Fe}_{60} \mathrm{Al}_{40}$ désordonné chimiquement et par suite ferromagnétique à la température ambiante.

L'évolution des propriétés magnétiques en fonction de la température, dans la gamme 293$1073 \mathrm{~K}$, a été également suivie par la technique CEMS. Après les recuits effectués dans les mêmes conditions que les échantillons déposés sur kapton, les spectres ont été réalisés à $293 \mathrm{~K}$. Ils sont représentés sur les figures $7 \mathrm{~b}$, c, d et leur dépouillement a conduit aux paramètres Mössbauer qui sont récapitulés dans le tableau II. Comme dans le cas du support de kapton, on constate que $\bar{H}$ et $f_{\mathrm{f}}$ croissent quand $T$ croît. En particulier, il est important de constater qu'à la température de $773 \mathrm{~K}$, la fraction ferromagnétique est de $100 \%$, c'est-à-dire qu'il n'y a plus de composante paramagnétique ; le champ hyperfin moyen est de $301 \mathrm{kOe}$, soit légèrement moins que le champ du fer $\alpha$ qui est égal à $330 \mathrm{kOe}$. Ce résultat confirme encore l'hypothèse de zones riches en fer. A la température de $873 \mathrm{~K}$, le spectre est constitué d'une seule raie paramagnétique; la raison la plus probable à ce changement d'allure pourrait être la diffusion des atomes de silicium appartenant au substrat dans le film. C'est pourquoi on a tenté de vérifier cette hypothèse en effectuant une étude SIMS sur l'échantillon qui a été recuit à $1073 \mathrm{~K}$. Celle-ci a conduit aux constatations suivantes (voir Fig. 9). Le traitement de recuit provoque l'homogénéisation de l'échantillon, sauf au voisinage de la surface où la pollution due au carbone est importante. De plus, le signal du silicium est relativement élevé

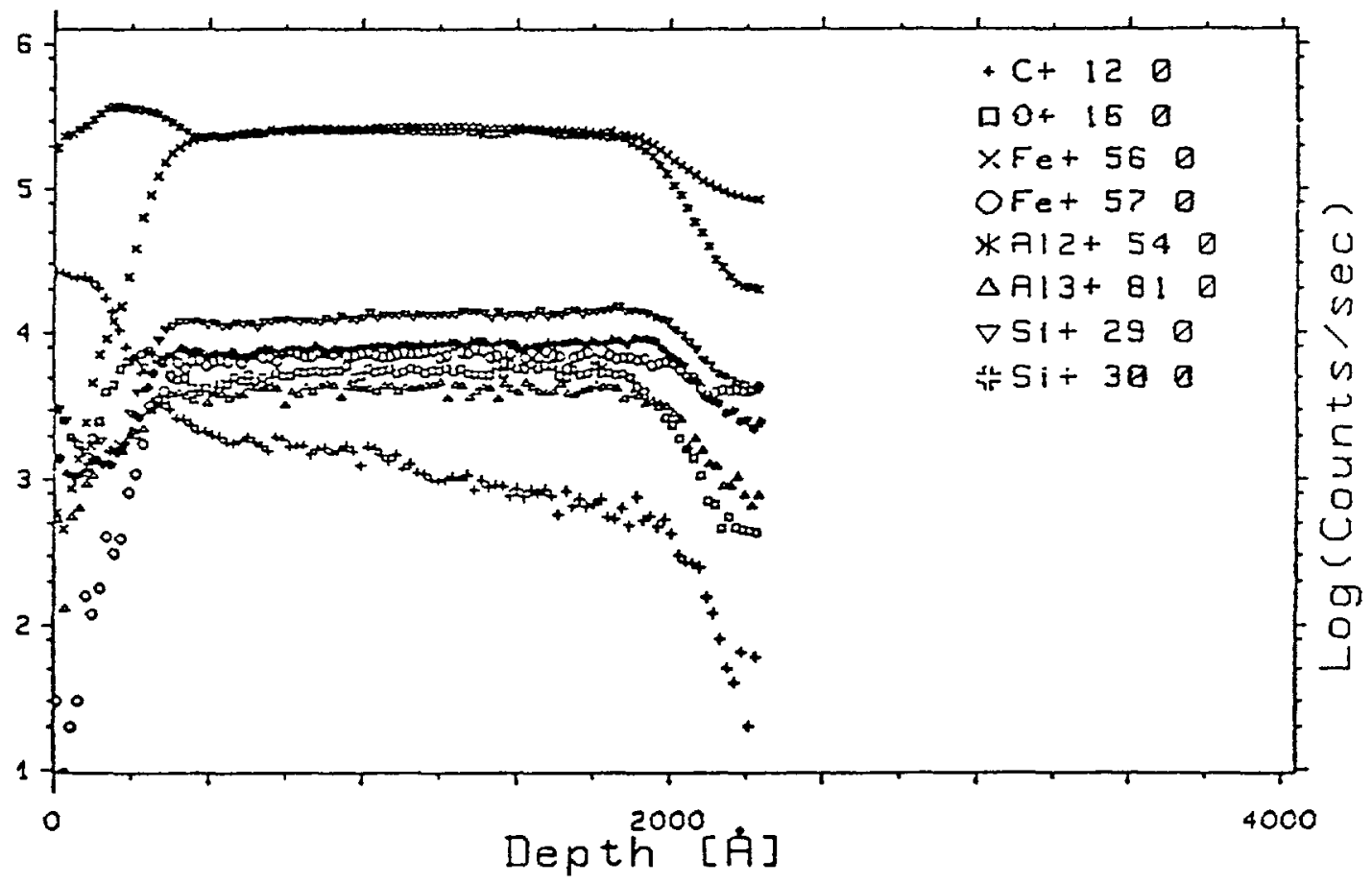

Fig. 9. - Profils SIMS de C, O, Fe, Al et Si dans le cas d'un film coévaporé de $200 \mathrm{~nm}$ déposé sur Si et recuit $1 \mathrm{~h}$ à $1073 \mathrm{~K}$.

[SIMS depth profiling of $\mathrm{C}, \mathrm{O}, \mathrm{Fe}, \mathrm{Al}$ and $\mathrm{Si}$ in the case of a coevaporated $200 \mathrm{~nm}$ film deposited onto $\mathrm{Si}$ and then annealed for one hour at $1073 \mathrm{~K}$.] 
et croît dans le domaine $20-100 \mathrm{~nm}$, zone qui devrait être exemptée de silicium s'il n'y avait pas diffusion. Cette deuxième observation conduit à montrer que la diffusion du silicium intervient sur la quasi-totalité de l'épaisseur du film.

\section{Propriétés magnétiques des films épais $\mathrm{Fe}_{60} \mathrm{Al}_{40}$.}

5.1 EFFET MÖSSBAUER EN TRANSMISSION. - Les spectres Mössbauer en transmission ont été réalisés sur des films d'épaisseur $3 \mu \mathrm{m}$ déposés sur kapton. Ils sont présentés sur la figure 10 et leur dépouillement a conduit aux paramètres Mössbauer indiqués dans le tableau III. Les spectres obtenus à $77 \mathrm{~K}$ et $293 \mathrm{~K}$ conduisent à des champs hyperfins moyens $\vec{H}$ de l'ordre de $200 \mathrm{kOe}$, ce qui est encore en bon accord avec la valeur du champ moyen obtenu sur les échantillons massifs implantés ou broyés. Il y a donc lieu de penser que dans le cas des films épais, les fluctuations de concentration - si elles existent - sont compensées dans l'épaisseur de l'échantillon. De plus, on peut affirmer encore que les trois modes de préparation des échantillons $\mathrm{Fe}_{60} \mathrm{Al}_{40}$ désordonnés donnent sensiblement le même résultat vis-à-vis du champ hyperfin moyen. Cependant, la fraction ferromagnétique $f_{\mathrm{f}}$ s'avère un peu plus petite que celle obtenue après implantation ( $47 \%$ au lieu de $67 \%$ ). Enfin, le spectre obtenu à $293 \mathrm{~K}$ sur l'échantillon recuit pendant une heure à $693 \mathrm{~K}$ permet de mesurer un champ moyen $\bar{H}=275 \mathrm{kOe}$, ce qui conduit encore à l'hypothèse de zones enrichies en fer.

Tableau III. - Paramètres Mössbauer des spectres en transmission, obtenus avec un film d'épaisseur $3 \mu \mathrm{m}$ déposé sur kapton. Les deux premiers spectres ont été obtenus à 77 et $293 \mathrm{~K}$ respectivement; le troisième a été réalisé à $293 \mathrm{~K}$ après recuit $1 \mathrm{~h}$ à $693 \mathrm{~K}$.

[Mössbauer parameters of transmission spectra taken from a $3 \mu \mathrm{m}$ thick film deposited onto a kapton substrate. The first two spectra were taken at 77 and $293 \mathrm{~K}$ respectively; the third one was taken at $293 \mathrm{~K}$ after 1 hour annealing at $693 \mathrm{~K}$.]

\begin{tabular}{|l|cccc|cc|}
\hline \multicolumn{1}{|c|}{$T(\mathrm{~K})$} & $\bar{H}(\mathrm{kOe})$ & $\sigma_{H}(\mathrm{kOe})$ & $b$ & $f_{\mathrm{f}}(\%)$ & $I S(\mathrm{~mm} / \mathrm{s})$ & $\Gamma(\mathrm{mm} / \mathrm{s})$ \\
\hline 77 & 205 & 49 & 1,1 & 59 & 0,15 & 0,50 \\
293 & 195 & 45 & 1,1 & 47 & 0,10 & 0,50 \\
après 1 h à 693 & 275 & 27 & 0,4 & 47 & 0,05 & 0,26 \\
\hline
\end{tabular}

Enfin, pour comparer les propriétés magnétiques de la surface à celles du cour du film, nous avons réalisé un spectre d'électrons à $293 \mathrm{~K}$ sur la surface d'un échantillon d'épaisseur $1 \mu \mathrm{m}$ déposé sur kapton; il est présenté sur la figure 11. En comparant ce spectre avec celui obtenu en géométrie de transmission (cf. Fig. 10b), on peut constater que la surface est légèrement différente du cœur du film. En effet, le spectre d'électrons conduit à un champ moyen de $203 \mathrm{kOe}$ et à une fraction ferromagnétique de $60 \%$, soit une augmentation de $20 \%$ de la fraction ferromagnétique et de $4 \%$ du champ moyen par rapport à l'échantillon épais.

5.2 Mesures d'Aimantation. - A la suite de l'étude des propriétés magnétiques locales, nous avons effectué des mesures d'aimantation sur les films épais. Contrairement à l'effet Kerr, le magnétomètre donne ici une information sur la totalité de l'échantillon. La figure 12 montre la courbe d'aimantation obtenue à $293 \mathrm{~K}$ sur un film d'épaisseur $1 \mu \mathrm{m}$ déposé sur kapton; le champ extérieur est ici appliqué parallèlement au film. On constate que la saturation est obtenue pour un champ appliqué de l'ordre de $1 \mathrm{kOe}$. La valeur de 


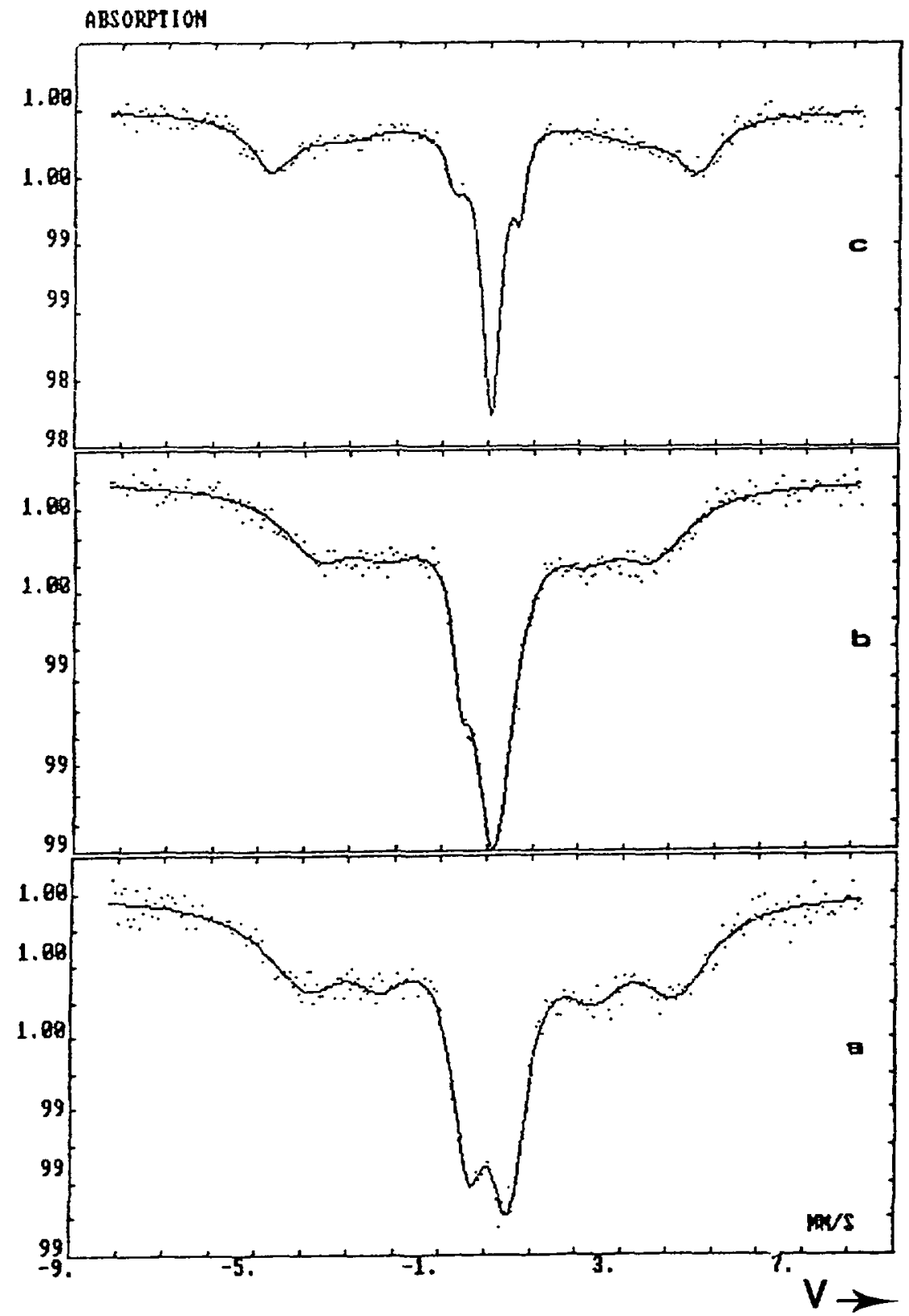

Fig. 10. - Spectres Mössbauer en transmission : a) film coévaporé $77 \mathrm{~K}$; b) $293 \mathrm{~K}$; c) recuit à $673 \mathrm{~K}$ et refroidi à la température ambiante.

[Transmission Mössbauer spectra : a) as-coevaporated film at $77 \mathrm{~K}$; b) $293 \mathrm{~K}$; c) annealed at $673 \mathrm{~K}$ and cooled down to room temperature.] 


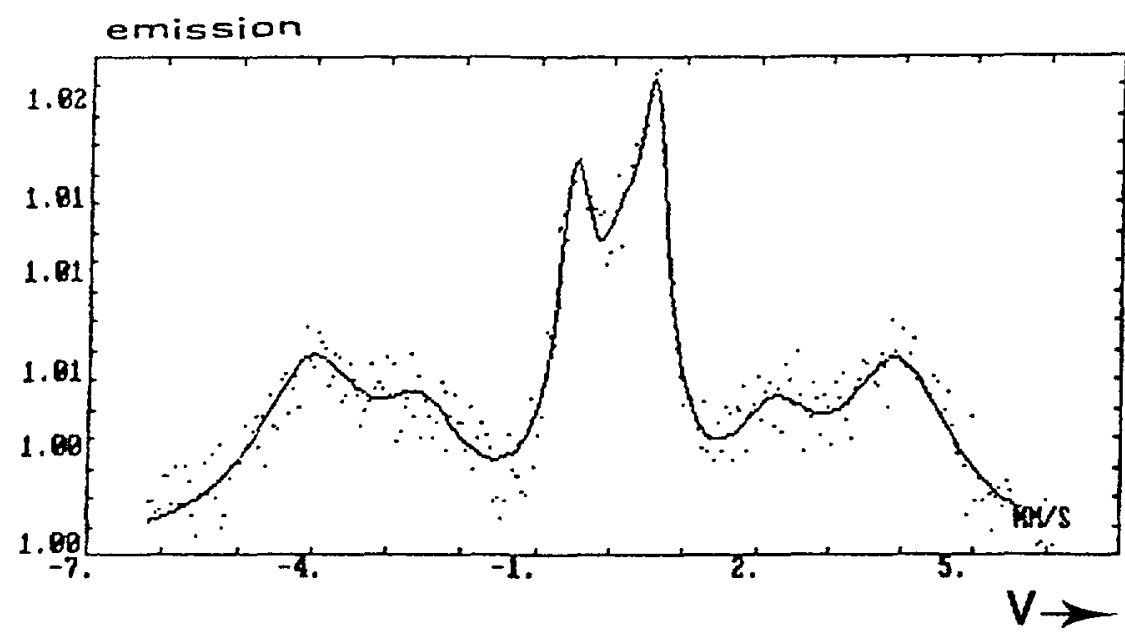

Fig. 11. - Spectre CEMS à $293 \mathrm{~K}$ de la surface d'un film d'épaisseur $1 \mu \mathrm{m}$ déposé sur kapton.

[Room temperature CEM spectrum taken from the surface of a $1 \mu \mathrm{m}$ thick film deposited onto a kapton substrate.]

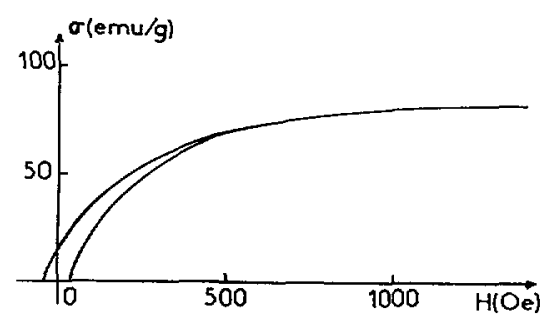

Fig. 12. - Courbe d'aimantation à $293 \mathrm{~K}$ en fonction du champ magnétique appliqué dans le plan d'un film d'épaisseur $1 \mu \mathrm{m}$ déposé sur kapton.

[Magnetization curve versus applied magnetic field at $293 \mathrm{~K}$ in the plane of a $1 \mu \mathrm{m}$ thick film deposited onto a kapton substrate.]

l'aimantation extrapolée à $H=0$ est de $85 \mathrm{uem} / \mathrm{g}$ et le champ coercitif est voisin de $40 \mathrm{kOe}$. Il est intéressant de noter que cette valeur est en bon accord avec celle obtenue par résonance ferromagnétique sur un échantillon $\mathrm{Fe}_{60} \mathrm{Al}_{40}$ massif implanté [12]. Enfin, la valeur de l'aimantation à saturation ainsi mesurée représente environ le tiers de celle du fer pur.

\section{Discussion et conclusion.}

La discussion portera essentiellement sur la comparaison entre les propriétés des échantillons $\mathrm{Fe}_{60} \mathrm{Al}_{40}$ coévaporés sur kapton ou sur silicium et les échantillons massifs implantés ou écrouis. Les résultats obtenus concernant le champ moyen $\bar{H}$ et la fraction ferromagnétique à $293 \mathrm{~K}$ sont récapitulés dans le tableau IV en fonction des différents modes d'élaboration des alliages $\mathrm{Fe}_{60} \mathrm{Al}_{40}$ désordonnés.

En ce qui concerne le film mince déposé sur kapton, le champ moyen est de $250 \mathrm{kOe}$ au lieu de $218 \mathrm{kOe}$ dans le cas du support de silicium. Cette différence peut être partiellement 
Tableau IV. - Champ hyperfin moyen $\bar{H}$ et fraction de fer ferromagnétique $f_{\mathrm{f}}$ en fonction du mode de préparation de l'alliage $\mathrm{Fe}_{60} \mathrm{Al}_{40}$ désordonné.

[Average hyperfine field $\bar{H}$ and percentage of ferromagnetic iron $f_{\mathrm{f}}$ for various preparation modes of disordered $\mathrm{Fe}_{60} \mathrm{Al}_{40}$ alloy.]

\begin{tabular}{|l|c|c|c|}
\hline \multicolumn{1}{|c|}{$\mathrm{Fe}_{60} \mathrm{Al}_{40}$} & $\tilde{H}(\mathrm{kOe})$ & $f_{\mathrm{f}}(\%)$ & $\begin{array}{c}\text { Références } \\
\text { bibliographiques }\end{array}$ \\
\hline Broyage du massif & 198 & 68 & {$[4]$} \\
\hline Implantation ionique du massif & 211 & 67 & {$[9]$} \\
\hline Film mince sur Si & 218 & 74 & cette étude \\
\hline Film épais sur kapton & 195 & 47 & cette étude \\
\hline Film mince sur kapton & 250 & 30 & {$[13]$} \\
\hline
\end{tabular}

interprétée par les fluctuations de concentration, en particulier celles observées par SIMS. La composante magnétique pourrait alors provenir de zones ayant $40 \%$ d'Al et aussi de zones ayant moins de $40 \%$. Cette interprétation peut aussi être étayée par de simples calculs, en particulier en utilisant la relation suivante qui lie le champ moyen au moment magnétique :

$$
H=[a+b(1-x)] / \mu_{\mathrm{Fe}}(x)
$$

où $a$ et $b$ sont des constantes valant 65 et $85 \mathrm{kOe} / \mu_{\mathrm{B}}$ respectivement et où $\mu_{\mathrm{Fe}}(x)$ est le moment magnétique d'un atome de fer dans l'alliage désordonné $\mathrm{Fe}_{1-x} \mathrm{Al}_{x}$ [10]. En prenant l'expression de $\mu_{\mathrm{Fe}}(x)$ donnée par Kouvel [16] :

$$
\mu_{\mathrm{Fe}}(x)=\mu_{\mathrm{Fe}}\left(1+0,14 x-0,16 x^{2}-3,120 x^{3}+2,67 x^{4}\right)
$$

on trouve $H=235$ kOe pour $x=0,35$ qui correspond à la limite inférieure du domaine B2 du diagramme d'équilibre $\mathrm{Fe}-\mathrm{Al}$; des valeurs de $x$ encore plus petites conduiraient naturellement à un champ moyen plus élevé, par exemple $x=0,30$ donne $H=255 \mathrm{kOe}$. L'hypothèse de fluctuations de concentration s'avère donc capable d'interpréter la valeur du champ moyen du film mince déposé sur kapton. Poursuivant la comparaison avec le matériau massif implanté, on peut aussi noter des différences dans la fraction de fer paramagnétique qui est de $70 \%$ pour l'échantillon déposé sur kapton au lieu de $30 \%$ après implantation du massif; ces différences sont certainement inhérentes aux modes de préparation des échantillons. Il y a donc lieu de penser que les dépôts sur silicium sont homogènes et par suite ne comportent pas de fluctuations de concentration de grande longueur d'onde. Enfin les spectres Mössbauer obtenus sur les films minces déposés sur kapton (ou sur silicium) montrent, par l'intermédiaire des raies $\Delta m=0$ que la polarisation des spins est principalement perpendiculaire au plan du film, contrairement au cas de l'alliage massif implanté (ou écroui) où les spins ont plutôt tendance à se polariser parallèlement à la surface de l'échantillon. Cette différence peut s'expliquer par la formation, dans le cas des films coévaporés, de structures colonnaires perpendiculaires au plan du film qui infléchiraient l'orientation des spins dans leur direction. En effet, dans les mêmes conditions d'évaporation, c'est-à-dire avec un angle d'incidence de 
la vapeur de $45^{\circ}$ et rotation du support, de telles structures colonnaires ont été observées par microscopie électronique à transmission dans des films de $\mathrm{Fe}$ et $\mathrm{Fe}_{60} \mathrm{Co}_{40}$ par Dinhut et Eymery [17]. Une telle microstructure a également été signalée dans des films du système Fe$\mathrm{Cu}$ obtenus en incidence oblique [18].

D'autre part, le comportement en température des films $\mathrm{Fe}_{60} \mathrm{Al}_{40}$ coévaporés s'est révélé être différent de celui de l'alliage massif implanté ou écroui. En effet, d'après les études rapportées ici, il apparaît clairement que l'alliage $\mathrm{Fe}_{60} \mathrm{Al}_{40}$ coévaporé ne se réordonne pas après chauffage au-dessus de la température ambiante; la solution solide préparée par coévaporation n'est donc certainement pas aléatoire. Ce résultat très important était cependant inattendu. Dans le cas de l'alliage massif implanté, les interactions magnétiques disparaissent à des températures sensiblement plus élevées que la température ambiante [11], ce qui conduit à l'ordre atomique B2 caractérisé par un spectre paramagnétique. Au contraire, le champ hyperfin des films coévaporés augmente avec la température de recuit jusqu'à $301 \mathrm{kOe}$; cette valeur est légèrement plus élevée que celle des sites $\mathrm{D}$ de l'alliage $\mathrm{Fe}_{3} \mathrm{Al}$ ordonné qui est de $290 \mathrm{kOe}$. Cette augmentation du champ indique la présence de zones enrichies en fer. Leur contribution au spectre s'accroît jusqu'à $100 \%$ à la température de $773 \mathrm{~K}$. La valeur correspondante du champ (301 kOe) indique qu'en moyenne un atome de fer est entouré de 7 atomes de fer premiers voisins. Il en résulte donc qu'à ces températures la diffusion est rendue possible, permettant ainsi la transformation de $\mathrm{Fe}_{60} \mathrm{Al}_{40}$ en une ou plusieurs phases de stabilité plus forte et plus proches de $\mathrm{Fe}_{3} \mathrm{Al}$ et du fer $\alpha$. Des résultats analogues ont été trouvés par Godbole et al. [19] dans des échantillons $\mathrm{Fe}_{55} \mathrm{Al}_{45}$ mixés par faisceaux d'ions sans toutefois que le problème de la mise en ordre atomique ait été soulevé. D'autres auteurs [20] ont montré, dans des multicouches Fe-Al mixées par des ions $\mathrm{Xe}^{+}$et recuites à différentes températures, la présence de phases cristallines stables ou métastables. Le comportement particulier des films vis-à-vis du massif peut être en partie attribué à des fluctuations locales de composition inhérentes au mode de préparation mais de période trop petite pour être décelable par les techniques d'analyse employées.

En conclusion, on a préparé des films de composition $\mathrm{Fe}_{60} \mathrm{Al}_{40}$ par coévaporation. Ceux-ci se sont avérés ferromagnétiques à la température ambiante et leurs propriétés apparaissent proches de celles du matériau massif correspondant désordonné par broyage ou par implantation ionique, à l'exception des films déposés sur kapton qui présentent quelques écarts. Cependant, dans la gamme de température $573-773 \mathrm{~K}$, les films montrent une tendance à la précipitation de phases nouvelles riches en fer, au lieu de l'ordre atomique B2 obtenu dans le cas de l'échantillon massif implanté. Un résultat important est donc que l'alliage $\mathrm{Fe}_{60} \mathrm{Al}_{40}$ préparé par coévaporation ne se réordonne pas.

\section{Remerciements.}

Les auteurs remercient les personnes qui ont apporté leur concours à la réalisation de cette étude: M. F. Denanot pour la microscopie électronique, M. Cahoreau et J. Caisso pour le SIMS, R. Krishnan (Bellevue) pour les mesures magnétiques. Il y a lieu de mentionner également que l'équipe de $P$. Imbert (Gif-sur-Yvette) nous a fourni les programmes Mössbauer utilisés.

\section{Bibliographie}

[1] Kuentzler R., J. Phys. France 44 (1983) 1167.

[2] Gengnagel H., Besnus M. J. et Danan H., Phys. Stat. Sol. A 13 (1972) 499.

[3] Besnus M. J., Herr A. et Meyer A. J. P., J. Phys. F. Metal Phys. 5 (1975) 2138. 
[4] Huffman G. P. et Fisher R. M., J. Appl. Phys. 38 (1967) 735.

[5] Wertheim G. K. et Wernick J. H., Acta Metall. 15 (1967) 297.

[6] Shiga M. et Nakamura Y., J. Phys. Soc. Jpn 405 (1976) 1295.

[7] OKi K., Towata S. et Eguchi T., Trans. Jpn Inst. Met. 22 (1981) 771.

[8] EyMery J. P., FNidiki A. et Riviere J. P., Nucl. Instr. Meth. 209-210 (1983) 919.

[9] Fnidiki A., Eymery J. P. et Delafond J., J. Magn. Magn. Mater. 40 (1983) 130.

[10] FNidiki A. et EYMERY J. P., Scr. Met. 19 (1985) 847.

[11] FNIDIKI A., Bodin D. et EYMERY J. P., Hyp. Inter. 29 (1986) 1179.

[12] Krishnan R., Suran G., Eymery J. P., Riviere J. P. et Fnidiki A., Phys. Lett. A 121 (1987) 43.

[13] Fnidiki A., EYmery J. P. et JunQUA N., Solid State Commun. 63 (1987) 549.

[14] Bodin D. et EyMery J. P., Nucl. Instr. Meth. B 16 (1986) 424.

[15] Window B., J. Phys. E 4 (1971) 401.

[16] Kouvel J. S., Magnetism and Metallurgy, vol. 2, edited by Berkowitz and Kneller (Academic Press, New York) 1969, p. 561.

[17] Dinhut J. F. et EYMERY J. P., à paraître dans J. Magn. Magn. Mater.

[18] KeItoko S., Kamimori T. et Goto M., Jpn J. Appl. Phys. 25 (1986) 1668.

[19] Godbole V. P., Chaudhari S. M., Chaisas S. V., Kanetka S. M. et Ogale S. B., Phys. Rev. B31 (1985) 5703.

[20] Raunschenbach B. et Hohmuth K., Nucl. Instr. Meth. B 23 (1987) 323. 